\begin{tabular}{|c|c|}
\hline Title & $\begin{array}{l}\text { Realistic Design of Large Hollow-Core Photonic Band-Gap Fibers With Suppressed Higher Order Modes and Surface } \\
\text { Modes }\end{array}$ \\
\hline Author(s) & Saitoh, Kunimasa; Florous, Nikolaos John; Murao, Tadashi; Koshiba, Masanori \\
\hline Citation & $\begin{array}{l}\text { Journal of Lightwave Technology, 25(9), 2440-2447 } \\
\text { https://doi.org/10.1109/LT .2007.902749 }\end{array}$ \\
\hline Issue Date & 2007-09 \\
\hline Doc URL & http:/hdl.handle.net/2115/30157 \\
\hline Rights & $\begin{array}{l}\text { O2007 IEEE. Personal use of this material is permitted. However, permission to reprint/republish this material for } \\
\text { advertising or promotional purposes or for creating new collective works for resale or redistribution to servers or lists, } \\
\text { or to reuse any copyrighted component of this work in other works must be obtained from the IEEE. }\end{array}$ \\
\hline Type & article \\
\hline File Information & JLT25-9.pdf \\
\hline
\end{tabular}

Instructions for use 


\title{
Realistic Design of Large-Hollow-Core Photonic Band-Gap Fibers With Suppressed Higher Order Modes and Surface Modes
}

\author{
Kunimasa Saitoh, Member, IEEE, Nikolaos John Florous, Member, IEEE, \\ Tadashi Murao, and Masanori Koshiba, Fellow, IEEE
}

\begin{abstract}
This paper theoretically describes effective suppression of higher order modes (HOMs) in realistic large-hollow-core photonic band-gap fibers (PBGFs) and utilizes the use of this class of waveguides for low-loss data-transmission applications and high-power beam delivery systems. The proposed design strategy is based on the resonant-coupling mechanism of central air-core modes with defected outer core modes. By incorporating six 7-unit-cell air cores in the cladding of the PBGF with sixfold symmetry, it is possible by resonantly coupling the light corresponding to the HOMs in a central 19-unit-cell core into the outer 7-unit-cell core, thus significantly increasing the leakage losses of the HOMs in comparison to those of fundamental mode. We consider a realistic PBGF structure with hexagonal airholes having rounded corners and derive a surface-mode-free condition of a silica-ring thickness surrounding the hollow core for both 7-unit-cell and 19-unit-cell cores. Verification regarding the propagation properties of the proposed design is ensured with a PBGF analysis based on a finite element modal solver. Numerical results show that the leakage losses of the HOMs can be enhanced in a level of at least three orders of magnitude over 200-nm wavelength range in comparison to those of the fundamental mode, while in addition, we show that the incorporation of a realistic air core with optimized silica-ring thickness can eliminate surface modes and achieve strong confinement into the central core and very low $\eta$-factor for the fundamental mode.
\end{abstract}

Index Terms-Finite-element method (FEM), high-power beam delivery, large mode area fibers, low attenuation, low nonlinearity, photonic band-gap fibers (PBGFs), single-mode fibers.

\section{INTRODUCTION}

$\mathbf{P}$ HOTONIC band-gap fibers (PBGFs) have recently attracted considerable attention and have shown great potentials in telecommunications and other application areas [1]. It has been conjectured that lightwave guidance through air cores via the photonic band-gap (PBG) effect, using a special category of microstructured optical fibers known as PBGFs [2], can revolutionize telecommunication industry, because the light can be controlled in the fiber in ways not previously possible.

Manuscript received January 23, 2007; revised April 26, 2007.

The authors are with the Division of Media and Network Technologies, Hokkaido University, Sapporo 060-0814, Japan (e-mail: ksaitoh@ist.hokudai. ac.jp; nflorous@dpo7.ice.eng.hokudai.ac.jp; murao@icp.ist.hoku.ac.jp; koshiba@ist.hokudai.ac.jp).

Color versions of one or more of the figures in this paper are available online at http://ieeexplore.ieee.org.

Digital Object Identifier 10.1109/JLT.2007.902749
Potential use of PBGFs becomes clearer when the unique properties of PBGFs can be directly related to the qualitatively novel functionalities. For example, the ability to guide light in a hollow core suggests a new technological regime of transmission with low attenuation [3], [4], high-power delivery with low nonlinearities [5]-[9], or nonlinear optics applications [10], [11].

Perhaps, the main technological issue that exists when guiding light through hollow-core PBGFs is the inevitable presence of higher order modes (HOMs), where, in the particular case of large cores, they exhibit leakage losses which are sometimes comparable to those of the fundamental mode. Although suppression of HOMs can be observed in small-core PBGFs [12], [13], there exists a fundamental limit of scattering losses due to the surface-roughness scattering [14], which prohibits further reduction of the fiber's attenuation to the level of the conventional fiber. One possible solution to overpass this limit is to consider large-hollow-core PBGFs, leading the multimode operation. In order to enhance the suppression of HOMs in microstructured optical fibers, an index-matching mechanism between the HOMs in the central core and the much leaky outer core modes or cladding modes around a certain wavelength range has been introduced [15]-[17], and recently, this intuitive idea has also been adapted to suppress the HOMs in hollowcore PBGFs [18] for effective single-mode operation. In our previous investigation, although the suppression of HOMs was possible, a hollow-core PBGF with circular airholes in the cladding and a nonrealistic core and cladding interface was considered [18]. However, in a practically fabricated hollowcore PBGF, the cladding airholes tend to be noncircular with a close-to-hexagonal shape at large air-filling fractions, and the hollow core also tends to be hexagonal with rounded corners [19], [20].

In this paper, we show that the suppression of HOMs in hollow-core PBGFs with realistic airholes in the cladding and core/cladding interface can be successfully achieved through the resonant-coupling mechanism of central air-core modes with defected outer core modes that we have proposed in [18]. Our primary target is derivation of a surface-mode-free condition for the silica-ring thickness surrounding the hollow core for both 7-unit-cell and 19-unit-cell air cores. Then, by incorporating six 7-unit-cell air cores, which are free of surface mode, in the cladding of the PBGF with sixfold symmetry, it is possible by resonantly coupling the light corresponding 


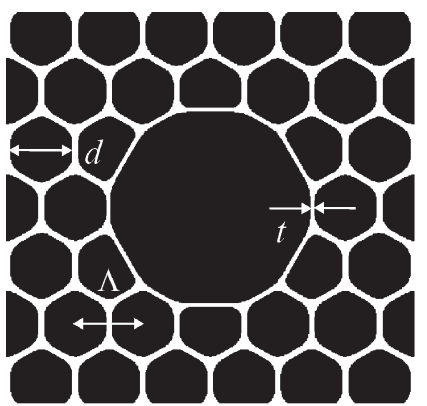

(a)

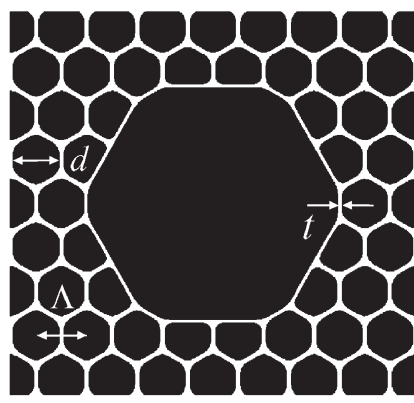

(b)
Fig. 1. Schematic cross sections of the PBGF profiles with air-hole diameters $d$ and lattice constant $\Lambda$, incorporating a silica ring with thickness $t$ surrounding the hollow core. (a) Corresponding PBGF with a 7-unit-cell air core and (b) PBGF with a 19-unit-cell air core.

to the HOMs in the central 19-unit-cell core into the outer 7-unit-cell core, thus significantly increasing the leakage losses of the HOMs in comparison to those of the fundamental mode. We perform numerical simulations using a modal solver based on the finite element method (FEM) [21], [22], and we show that the leakage losses of the HOMs can be enhanced in a level of at least three orders of magnitude over 200-nm wavelength range in comparison to those of the fundamental mode, while in addition, we show that the incorporation of a realistic hollow core with the optimized silica-ring thickness can achieve strong confinement into the central core without surface mode and very low $\eta$-factor for the fundamental mode even in the case that the core-surrounding silica-ring thickness does not satisfy an antiresonant condition [4].

\section{SupPrESSION OF SURFACE MOdES IN LARGE-HOLLOW-CORE PBGFS}

Since the motivation of our investigation is to design realistic large-hollow-core effective single-mode PBGFs with a broadband transmission, we have to appropriately determine the fiber's structural parameters. In order to obtain large PBG in the cladding, we need a very high air-filling fraction, which leads the cladding airholes to tend to be hexagonal in shape with rounded corners, as shown in Fig. 1. In Fig. 1(a), we model an idealized but realistic standard 7-unit-cell hollowcore PBGF, where the cladding holes were represented by hexagons with rounded corners described by the relative hole diameter $d / \Lambda$ and the relative diameter of curvature at the corners $d_{\mathrm{c}} / \Lambda$ [23], [24] with $\Lambda$ as the hole pitch. The corners of the pentagons surrounding the hollow core are rounded using circles of relative diameter $d_{c}^{\prime} / \Lambda$ [24]. The hexagonal core with rounded corners is defined by a thin silica ring of nearly constant thickness $t$ at the boundary of the cladding [20]. The 7-unit-cell hollow-core size is not so large, and therefore, such core design makes it difficult to suppress the scattering losses due to the surface roughness [4]. In order to form a larger hollow-core PBGF, we can remove three rows of tubes, and the resulting structure is shown in Fig. 1(b). We will refer to this type of PBGF as a PBGF with a 19-unit-cell core. In a fabricated hollow-core PBGF with quite similar fiber cross

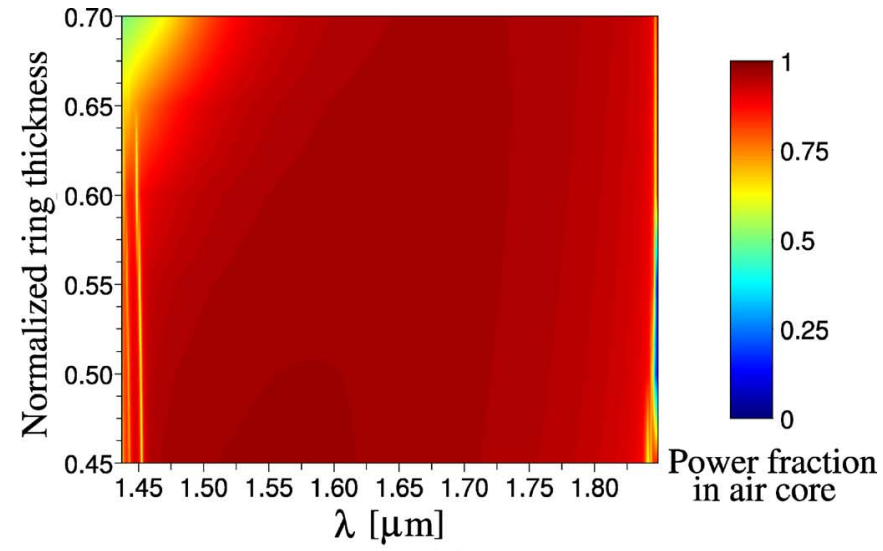

(a)

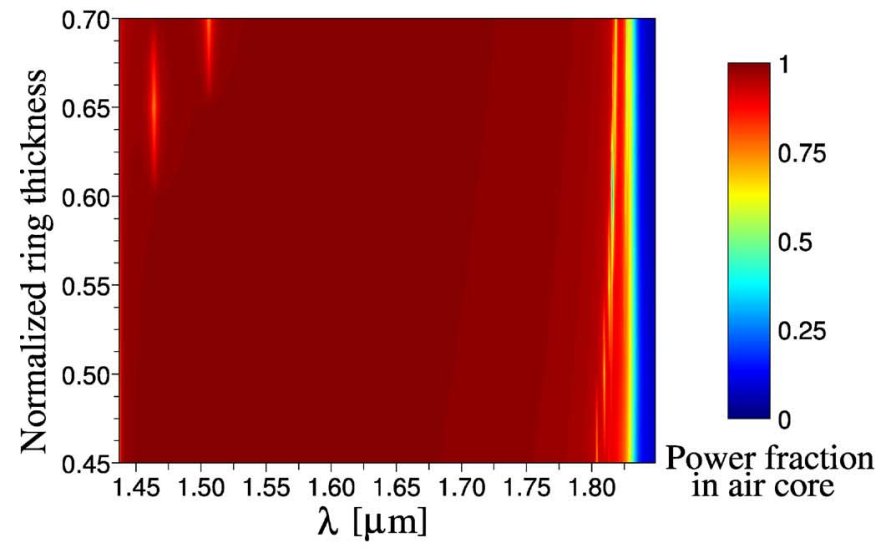

(b)

Fig. 2. Color map of the power fraction in the air core as a function of the normalized silica-ring thickness and the operating wavelength for (a) 7-unitcell air-core PBGF and (b) 19-unit-cell air-core PBGF, where $d / \Lambda=0.97$, $d_{\mathrm{c}} / \Lambda=0.55, d_{\mathrm{c}}^{\prime} / \Lambda=0.317$, and $\Lambda=4.0 \mu \mathrm{m}$.

section shown in Fig. 1, in general, not only air-guiding modes which concentrate most of their energy in the air-core region but also surface modes which localize energy at the core-cladding interface are supported. It is known that the coupling between the air-guiding mode and the surface mode is a major source of propagation loss in hollow-core PBGF [25] and this coupling, which results in the reduction of the transmission bandwidth, making the presence of surface modes the limitation for the development of hollow-core PBGF with low attenuation and a broadband transmission spectrum [20]. Recently, AmezcuaCorrea et al. have reported that elimination of the surface modes can be achieved by optimizing the silica-ring thickness [20]; however, the silica-ring thickness has been optimized only for the 7-unit-cell PBGF shown in Fig. 1(a), with a particular value of $d / \Lambda=0.95$. Here, we increase the airhole diameter up to $d / \Lambda=0.97$ in the cladding to obtain broader transmission spectrum and to optimize the silica-ring thickness not only for the 7-unit-cell PBGF but also the 19-unit-cell PBGF.

For the structures shown in Fig. 1(a) and (b), in order to study the impact of the silica ring surrounding the hollow core, in conjunction with the suppression of the surface modes in Fig. 2, we show a contour color-map representation of the power fraction in the air core as a function of the normalized silicaring thickness and the operating wavelength, for the following: 
1) the 7-unit-cell hollow-core PBGF and 2) the 19-unit-cell hollow-core PBGF. The design parameters were chosen as follows: relative airhole diameters $d / \Lambda=0.97, d_{\mathrm{c}} / \Lambda=0.55$ [20], and $d_{\mathrm{c}}^{\prime} / \Lambda=0.317$ [20], and the normalized silica-ring thickness $T$ is varied in the range $0.45 \leq T \leq 0.70$, where the normalized silica-ring thickness is defined as [20]

$$
T=t /(\Lambda-d)
$$

The hole pitch $\Lambda=4 \mu \mathrm{m}$ was chosen to obtain a band gap centered around 1550-nm wavelength range. In Fig. 2, red color corresponds to regions where the fundamental air-guiding mode is strongly confined in the hollow core, while yellow color corresponds to coupling between the fundamental airguiding mode with surface modes or cladding modes, and the blue color corresponds to outside the PBG region. From these results, we can clearly see that, for both cases of 7-unit-cell and 19-unit-cell hollow-core PBGFs, we may find a wide range of normalized silica-ring thickness and operating wavelengths within which the power fraction of light guided into the air core is almost $100 \%$ over a wavelength range without any surface modes, and we can conclude that the optimized normalized silica-ring thickness is $T \approx 0.5$ for both the 7-unit-cell and 19-unit-cell PBGFs, as reported in [20].

\section{SUPPRESSION OF HOMS IN LARGE-HOLLOW-CORE PBGFS}

For the structures in Fig. 1, the effective-refractive-index curves as a function of the operational wavelength $\lambda$ are shown in Fig. 3, which are computed through a full-vector FEM modal solver [21], where the normalized airhole diameters $d / \Lambda=0.97, d_{\mathrm{c}} / \Lambda=0.55$, and $d_{\mathrm{c}}^{\prime} / \Lambda=0.317$, the hole pitch $\Lambda=4.0 \mu \mathrm{m}$, and the silica-ring thickness $t=0.5(\Lambda-d)$ to prevent the existence of surface modes derived in the previous section, and the refractive indexes of air and silica are 1.0 and 1.45 , respectively. The air-filling fraction $f$ of the cladding structure is given by

$$
f=\left(\frac{d}{\Lambda}\right)^{2}\left\{1-\left(1-\frac{\pi}{2 \sqrt{3}}\right)\left(\frac{d_{\mathrm{c}}}{d}\right)^{2}\right\}
$$

The PBGF with $d / \Lambda=0.97$ and $d_{\mathrm{c}} / \Lambda=0.55$ has $f \approx 0.913$. In particular, Fig. 3(a) shows the computed effective-refractiveindex curve of the fundamental mode in the 7-unit-cell PBGF shown in Fig. 1(a), and Fig. 3(b) shows the effective-refractiveindex curves of the fundamental mode in the 19-unit-cell PBGF shown in Fig. 1(b) as well as the HOMs of $\mathrm{TE}_{01}$-like mode, $\mathrm{TM}_{01}$-like mode, and the degenerated $\mathrm{HE}_{21}$-like mode, where the shaded regions represent PBG boundaries calculated by FEM. In the 19-unit-cell PBGF, additional HOMs (the $\mathrm{EH}_{11^{-}}$ like and $\mathrm{HE}_{31}$-like modes) also exist; however, their leakage losses are sufficiently large, so we do not need to try to enhance further their leakage losses. From the results in Fig. 3, we can clearly see that the curve corresponding to the fundamental mode in the small-core PBGF is quite close to the curves

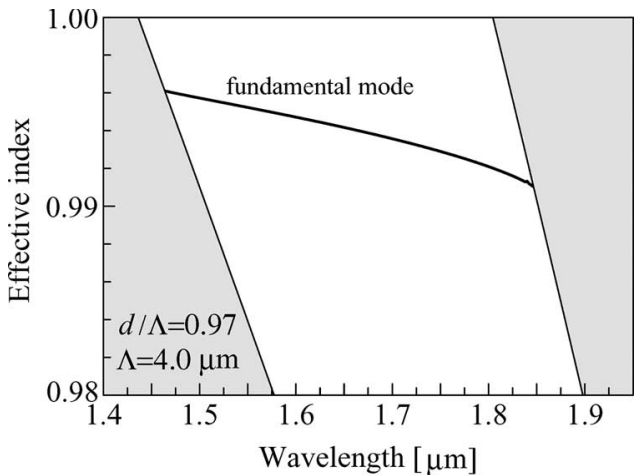

(a)

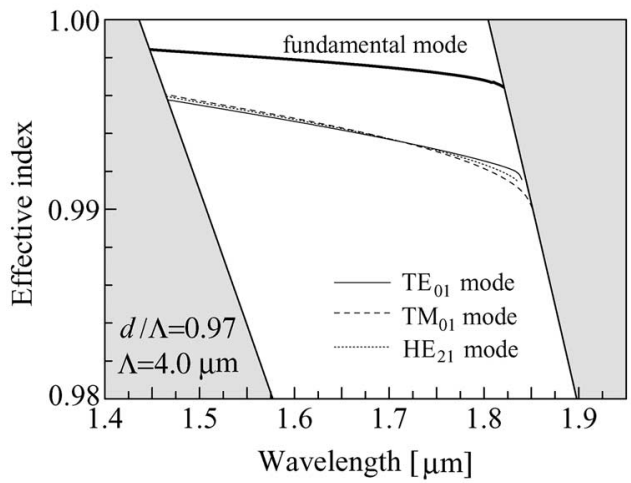

(b)

Fig. 3. Effective index curves as a function of the wavelength for (a) PBGF with 7-unit-cell core and (b) PBGF with 19-unit-cell core, where $d / \Lambda=0.97$, $d_{\mathrm{c}} / \Lambda=0.55, d_{\mathrm{c}}^{\prime} / \Lambda=0.317, t=0.5(\Lambda-d)$, and $\Lambda=4.0 \mu \mathrm{m}$, with the gray strips representing the PBG boundaries.

corresponding to the effective refractive indexes of the HOMs in the large-core PBGF. This fact can enable the index-matching mechanism to take place by bringing the two cores (19-unit-cell central core and 7-unit-cell outer core) close to each other, thus enabling resonant coupling over a certain wavelength regime. The phenomenon is expected to be enhanced further and to be a polarization-independent operation if we place more outer cores while keeping the sixfold symmetry around the central core in the PBGF as proposed in [18].

In order to enable the index-matching mechanism to occur between the HOM in 19-unit-cell core and the fundamental mode in 7-unit-cell core, we proceed by incorporating six 7 -unit-cell cores into the cladding surrounding the central 19-unit-cell core to expect a polarization-independent operation, as shown in Fig. 4. Introducing several outer cores close to the central core would increase the leakage losses of the HOMs in comparison to those of the fundamental mode. In such structure, although HOMs still exist in the central core, we can talk for an effective single-mode operation over a certain wavelength window. In this case, there is only one airhole between the two adjacent cores, since we want to obtain a strong coupling (such a strong coupling cannot be achieved when using more periods to isolate the two cores). If the resonant coupling occurs at a particular wavelength, an avoided crossing event of the guided modes causes a resonant peak with a Lorentzian shape in the leakage-loss spectrum. However, a strong coupling between the fundamental $\mathrm{HE}_{11}$-like mode in 


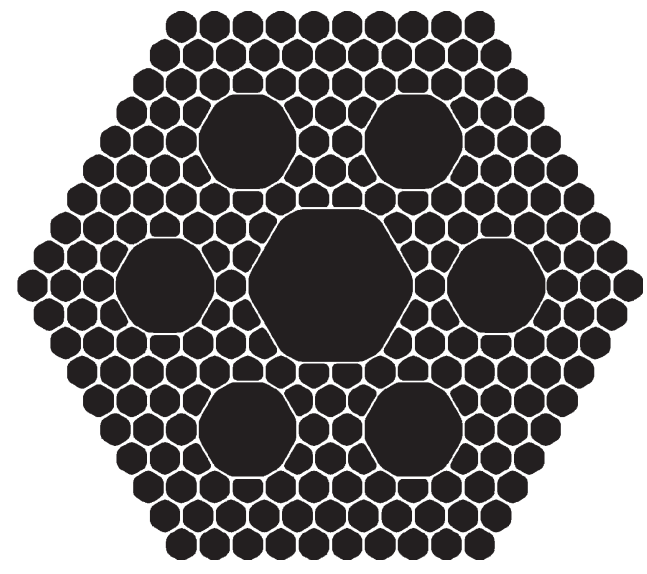

Fig. 4. Schematic cross section of the realistic large-hollow-core PBGF profile with a sixfold symmetric distribution of outer cores in the cladding for effective single-mode operation. The index-matching mechanism can enable the resonant coupling of the HOMs of the central core to the outer cores for HOMs suppression, while the optimized silica-ring thickness surrounding the air cores can eliminate the surface modes.

the 7-unit-cell core and the HOMs in the 19-unit-cell core can be expected for a broad wavelength region due to the fact that the effective index of the fundamental mode in the 7-unit-core PBGF is quite close to that of the HOMs in the 19-unit-core PBGF over the entire PBG range. Such resonant peaks in the leakage-loss spectrum will not appear. Therefore, broadband leakage-loss enhancement can be expected in the PBGFs, as shown in Fig. 4. This is an advantage of our proposed structure, to obtain effective single-mode operation over a wide wavelength range.

Fig. 5 shows the computed leakage-loss properties of the structure in Fig. 4 as a function of the operational wavelength by using a FEM modal solver incorporating anisotropic perfectly matched layers as absorbing boundary conditions to evaluate the leakage losses described in detail in [21] and the references therein. Here, we briefly list the most important definitions. For a given frequency $\omega=c k=c 2 \pi / \lambda$ (where $c$ is velocity of light and $k$ and $\lambda$ are the free-space wavenumber and wavelength, respectively), numerical calculation provides us with a complex propagation constant $\gamma(\omega)=\beta(\omega)+i \alpha(\omega)$ where $\beta$ is the usual propagation constant component along the fiber axis associated with the mode, and $\alpha$ is the attenuation constant associated with the exponential decay along the fiber axis. We present the attenuation on a decibel-scale by $20 \times$ $\log _{10}(e) \times \alpha \cong 8.686 \times \alpha$. In particular, Fig. 5(a) shows the evaluation of the leakage losses for the original 19-unit-cell hollow-core PBGF without defected cores and Fig. 5(b) with defected cores in the cladding. The various curves correspond to different modes, namely, the fundamental $\mathrm{HE}_{11}$-like mode (solid thick curve), the higher order $\mathrm{TE}_{01}$-like mode (solid thin curves), the higher order $\mathrm{TM}_{01}$-like mode (dashed curves), and the degenerated higher order $\mathrm{HE}_{21}$-like mode (dotted curves). The computation has been performed for the following set of design parameters: $d / \Lambda=0.97, d_{\mathrm{c}} / \Lambda=0.55, d_{\mathrm{c}}^{\prime} / \Lambda=0.317$, $\Lambda=4.0 \mu \mathrm{m}, t=0.5(\Lambda-d)$, and the total number of airhole rings counted from the edge of the central core to the outermost airhole ring is seven. Regarding the leakage losses for the

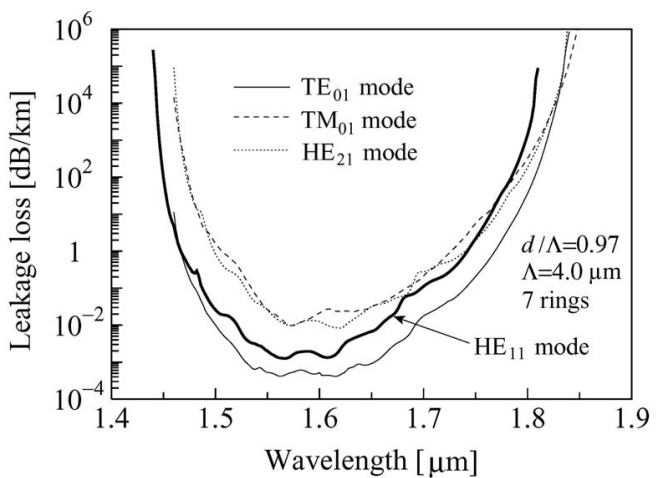

(a)

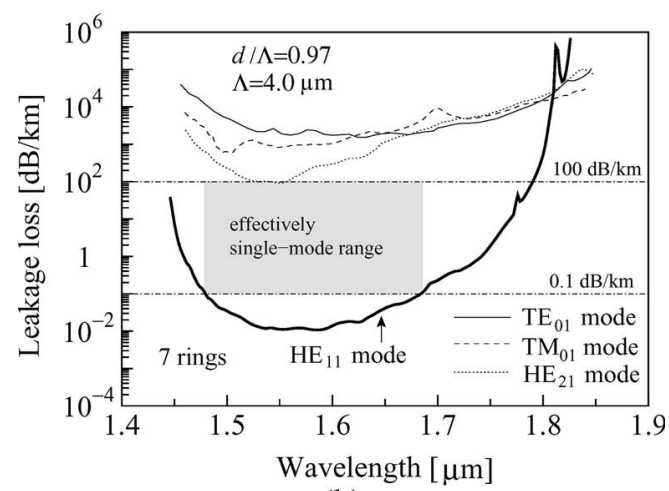

(b)

Fig. 5. (a) Leakage losses in decibels per kilometer of the fundamental mode (solid thick curve) and the HOMs (thin solid/dashed/dotted curves) as a function of the operational wavelength for the 19-unit-cell hollow-core PBGF without outer cores and (b) in the presence of outer cores. Observe the effective enhancement of the losses of the HOMs in the wavelength range corresponding to the effective single-mode range (gray box), in comparison to the fundamental mode.

19-unit-cell hollow-core PBGF without defected outer cores in the cladding, we can clearly observe that the leakage losses corresponding to the higher order $\mathrm{TE}_{01}$-like mode are comparable to those of the fundamental $\mathrm{HE}_{11}$-like mode and that the difference of the leakage losses between the fundamental mode and the HOMs of $\mathrm{TM}_{01}$-like and $\mathrm{HE}_{21}$-like mode is less than one order of magnitude, whereas the 19-unit-cell hollow-core PBGF with outer cores exhibits an effective single-mode operation from 1.48 - to $1.68-\mu \mathrm{m}$ wavelength range (namely over a 200-nm bandwidth), with the leakage-loss level of the fundamental mode to remain below $0.1 \mathrm{~dB} / \mathrm{km}$. As shown in Fig. 5(b), the leakage-loss level of the HOMs stays above $100 \mathrm{~dB} / \mathrm{km}$, leading to the suppression of HOMs. Therefore, the leakage losses of the HOMs have been enhanced by three orders of magnitude over a 200-nm wavelength window. Note that the destruction of the perfect periodic structure in the cladding caused by the existence of the outer cores also increases the leakage loss of the air-core modes to some extent. However, this contribution is not significantly large, because the difference of the leakage loss of the fundamental $\mathrm{HE}_{11}$-like mode with and without outer cores is small (approximately one order of magnitude) as shown in Fig. 5. On the other hand, the difference of the leakage loss of the HOMs in these two structures is very large (more than four orders of magnitude). From these results, we can conclude that the enhancement of the leakage 


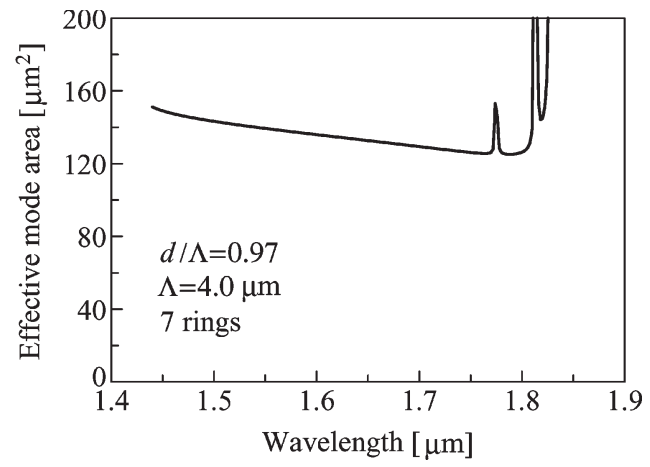

Fig. 6. Wavelength dependence of the effective mode area of the proposed 19-unit-cell hollow-core PBGF with outer cores, where $d / \Lambda=0.97, d_{\mathrm{c}} / \Lambda=$ $0.55, d_{\mathrm{c}}^{\prime} / \Lambda=0.317, t=0.5(\Lambda-d)$, and $\Lambda=4.0 \mu \mathrm{m}$.

losses of HOMs comes from the strong coupling between the central large core and the adjacent outer cores. It is difficult to point out the critical value which causes an appropriate index matching enough to effectively enhance the leakage loss of the HOMs, because this value is strongly dependent on the coupling coefficient between the HOMs and the outer core modes and the leakage-loss level of the HOMs of the PBGFs without the outer cores. However, we can say that the effective index difference between the fundamental $\mathrm{HE}_{11}$-like mode in a small hollow core and the HOMs in a large hollow core should be less than $10^{-3}$ in the proposed PBGFs. In Fig. 5, we have presented the leakage loss as a function of the wavelength for the hollow-core PBGF that has $d / \Lambda=0.97$. In order to derive the structural parameter of $d / \Lambda=0.97$, the $d / \Lambda$ was varied from 0.95 to 0.98 , and we have confirmed that, in every case, the leakage loss of the HOMs can be enhanced to some extent by the resonant coupling. For smaller values of $d / \Lambda(<0.96)$, additional airhole rings are required to lower the leakage loss of the fundamental mode below $0.1 \mathrm{~dB} / \mathrm{km}$ or less. In addition, for larger values of $d / \Lambda(>0.98)$, the PBG range can be widened, but the leakage-loss level of the HOMs cannot be enhanced up to $100 \mathrm{~dB} / \mathrm{km}$. Based on these facts, we can say that $d / \Lambda=0.97$ is the best parameter choice for the proposed PBGFs. We have chosen $d_{\mathrm{c}} / \Lambda=0.55$ as the most common value, as mentioned in [20], since $d_{c} / \Lambda$ does not depend significantly on the fiber properties such as effective indexes, leakage-loss level, etc.

In order to ensure that the proposed structure in Fig. 4 can be successfully used as a data transmission or high-power delivery system, a very crucial quantity is the available effective mode area of the structure. In Fig. 6, we show the calculated effective mode area in square micrometer as a function of the operating wavelength for the proposed 19-unit-cell hollow-core PBGF. Regarding the effective mode area of the fiber core $A_{\text {eff }}$, we use the following definition:

$$
A_{\mathrm{eff}}=\frac{\left(\iint_{s}|\boldsymbol{E}|^{2} d x d y\right)^{2}}{\iint_{s}|\boldsymbol{E}|^{4} d x d y}
$$

where $\boldsymbol{E}$ is the electric field vector, and $S$ denotes the whole fiber cross section. As can be clearly seen from the result, the effective mode area remains above $130 \mu \mathrm{m}^{2}$ over $200-\mathrm{nm}$

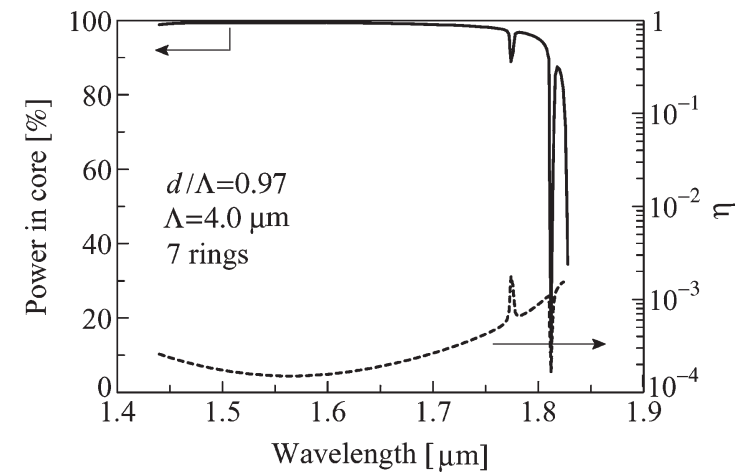

Fig. 7. Wavelength dependence of the (solid curve, left axis) power fraction in the core and the (dashed curve, right axis) $\eta$-factor in the proposed 19-unitcell hollow-core PBGF, where $d / \Lambda=0.97, d_{\mathrm{c}} / \Lambda=0.55, d_{\mathrm{c}}^{\prime} / \Lambda=0.317$, $t=0.5(\Lambda-d)$, and $\Lambda=4.0 \mu \mathrm{m}$.

wavelength band. The effective mode area in this case is a little bit larger than that of a conventional single-mode fiber, thus enabling the potential use of the proposed structure as a platform for information transmission with low loss and low nonlinearity or for high-power beam delivery. The sharp peak which occurs around the operating wavelength of $\lambda=1.774 \mu \mathrm{m}$ is associated with the coupling of the fundamental mode to the surface mode that is newly generated by the different condition from PBGFs without outer cores. In other words, the surface mode occurs between the silica ring of the inner core and that of the outer cores. The sharp peak around $\lambda=1.813 \mu \mathrm{m}$ is associated with the coupling of the fundamental mode to the cladding modes. However, using the optimized silica-ring thickness, there are no such coupling events inside low-loss transmission range, namely, from 1.48 - to $1.68-\mu \mathrm{m}$ wavelength.

When the field intensity at the glass/air interface or light-inglass power fraction is increased, the scattering losses due to the roughness of the silica-ring surface are enlarged [4], [14]. To avoid increasing the power in the silica ring surrounding the core, Roberts et al. [4] proposed to design the width of the silica ring around the core to satisfy the antiresonant condition [26], [27] at the midgap wavelength, in addition to the enlargement of the core radius, by removing 19 airholes to form the core [14]. This structure is one of the candidates for the realization of ultimate low-loss PBGFs, with the lowest limitation of the loss that has been estimated in [14]. However, the loss reduction in the structure has a consequence to reduce the available operational bandwidth because of the large number of anticrossing events between air-core and surface modes. If we can push the anticrossing events between air-core and surface modes out of the PBG range, the dominant factor of loss in hollow-core PBGFs is the scattering loss due to the roughness of the silica-ring surface, and this loss is proportional to the value of $\eta$-factor [4], where the $\eta$-factor is defined as the normalized overlap integration of the power in the silica ring as follows:

$$
\eta=\left|\frac{\int_{\text {glass annulus }} d A \boldsymbol{E} \times \boldsymbol{H}^{*} \cdot \boldsymbol{z}}{\int_{\text {cross section }} d A \boldsymbol{E} \times \boldsymbol{H}^{*} \cdot \boldsymbol{z}}\right|
$$




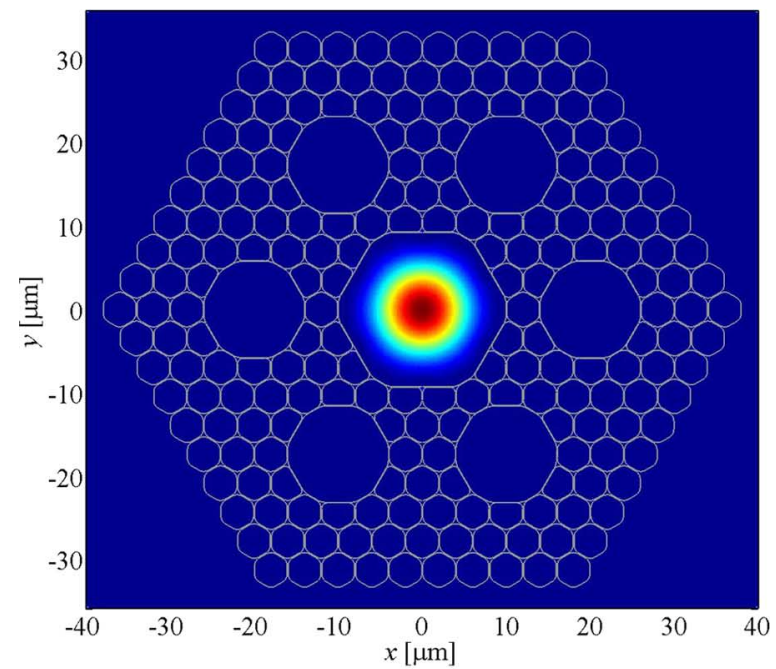

(a)

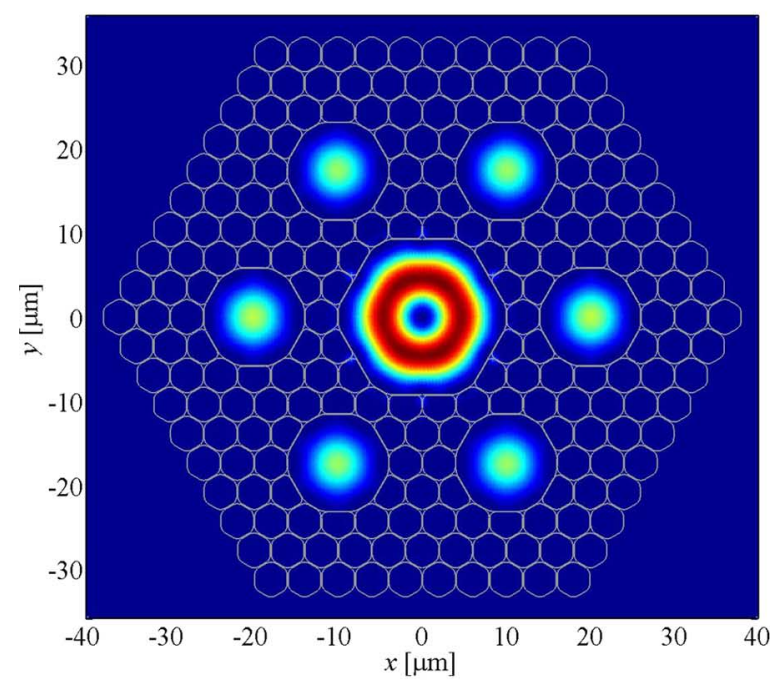

(b)

Fig. 8. (a) $z$-component of the Poynting vector of the fundamental $\mathrm{HE}_{11-}$ like mode at a wavelength of $\lambda=1.55 \mu \mathrm{m}$ and (b) higher order $\mathrm{TM}_{01}$-like mode at a wavelength of $\lambda=1.60 \mu \mathrm{m}$ in the proposed 19-unit-cell hollow-core PBGF, where $d / \Lambda=0.97, d_{\mathrm{c}} / \Lambda=0.55, d_{\mathrm{c}}^{\prime} / \Lambda=0.317, t=0.5(\Lambda-d)$, and $\Lambda=4.0 \mu \mathrm{m}$.

where $\boldsymbol{E}$ and $\boldsymbol{H}$ are the electric and magnetic fields of the fundamental mode, respectively, and $z$ is the unit vector along the fiber axis. Fig. 7 shows the wavelength dependence of the power fraction in the air core (solid curve) and the $\eta$-factor (dashed curve) for the proposed 19-unit-cell hollow-core PBGF. We can clearly see the inverse relation between the power fraction in the air core and the $\eta$-factor. The fundamental mode is tightly confined in the hollow-core region, and the percentage of the power in the core was found to be larger than $99 \%$ from 1.48to $1.68-\mu \mathrm{m}$ wavelength. In addition, due to its large core radius, a very low $\eta$-factor was obtained, which leads to the guidance of light with low attenuation. We note that the lowest $\eta$-factor attained for this fiber is $1.5 \times 10^{-4}$ at $1.56-\mu \mathrm{m}$ wavelength, which is one order of magnitude lower than the minimum found in the 19-unit-cell air-core PBGFs with an antiresonant core surround [4], and that this $\eta$-factor is the lowest value for singlemode hollow-core PBGFs reported so far.
In order to visualize the index-matching phenomenon which gives rise to the strong coupling of the HOMs in the central core to the outer cores, and which is responsible for the effective single-mode operation of the proposed structure, in Fig. 8(a), we plot the $z$-component of the Poynting vector of the fundamental $\mathrm{HE}_{11}$-like mode at a wavelength of $\lambda=1.55 \mu \mathrm{m}$, while in Fig. 8(b), we show the $z$-component of the Poynting vector of the higher order $\mathrm{TM}_{01}$-like mode at a wavelength of $\lambda=1.60 \mu \mathrm{m}$. It is evident that the fundamental mode is confined nicely into the air core of the fiber; on the other hand, there is an obvious coupling of the HOMs into the much leaky outer core modes. If the outer cores are absent in the cladding, such coupling does not occur, and the HOM is confined into the central core strongly.

\section{CONCLUSION}

To conclude the present investigation, we have shown that, with a particular engineering of the cladding in PBGFs, incorporating resonant-guiding structures, we can achieve selective suppression of unwanted modes in a large-hollow-core PBGF. If sufficient suppression of unwanted modes is achieved, the fiber can be considered effectively single-moded, and critical transmission impairments can be avoided. More interestingly, suppressing HOMs can actually reduce the fundamental loss limit by relaxing a practical constraint on the core size and, thus, the interaction of transmitted light with surface irregularities. HOM-suppressed designs are then one strategy for improving the fundamental loss limit, which is a key obstacle to using air-core fibers for transmission. In addition, the incorporation of a realistic air core with an optimized silica-ring thickness can eliminate the surface modes and can achieve strong confinement into the central core and very low $\eta$-factor for the fundamental mode. Extensive numerical simulations presented here show that significant suppression of HOMs is possible for fibers similar to those used in the lowest loss air-core simulations and experiments. Our proposed structure exhibits the following propagation characteristics: effective single-mode operation from 1.48 - to $1.68-\mu \mathrm{m}$ wavelength range with the leakage losses of the HOMs to be enhanced up to a level of $(100 \mathrm{~dB} / \mathrm{km})$ in comparison to the fundamental mode $(0.1 \mathrm{~dB} / \mathrm{km})$, low $\eta$-factor level of $1.5 \times 10^{-4}$, and an effective mode area of about $130 \mu \mathrm{m}^{2}$.

\section{REFERENCES}

[1] P. S. J. Russell, "Photonic crystal fibers," Science, vol. 299, no. 5605, pp. 358-362, Jan. 2003.

[2] R. F. Cregan, B. J. Mangan, J. C. Knight, T. A. Birks, P. S. J. Russell, P. J. Roberts, and D. C. Allan, "Single-mode photonic band gap guidance of light in air," Science, vol. 285, no. 5433, pp. 1537-1539, Sep. 1999.

[3] J. Shephard, W. MacPherson, R. Maier, J. Jones, D. Hand, M. Mohebbi, A. George, P. Roberts, and J. Knight, "Single-mode mid-IR guidance in a hollow-core photonic crystal fiber," Opt. Express, vol. 13, no. 18, pp. 7139-7144, Sep. 2005.

[4] P. Roberts, D. Williams, B. Mangan, H. Sabert, F. Couny, W. Wadsworth, T. Birks, J. Knight, and P. Russell, "Realizing low loss air core photonic crystal fibers by exploiting an antiresonant core surround," Opt. Express, vol. 13, no. 20, pp. 8277-8285, Oct. 2005.

[5] J. Shephard, J. Jones, D. Hand, G. Bouwmans, J. Knight, P. Russell, and B. Mangan, "High energy nanosecond laser pulses delivered single-mode through hollow-core PBG fibers," Opt. Express, vol. 12, no. 4, pp. 717723, Feb. 2004 
[6] G. Humbert, J. Knight, G. Bouwmans, P. Russell, D. Williams, P. Roberts, and B. Mangan, "Hollow core photonic crystal fibers for beam delivery," Opt. Express, vol. 12, no. 8, pp. 1477-1484, Apr. 2004.

[7] W. Göbel, A. Nimmerjahn, and F. Helmchen, "Distortion-free delivery of nanojoule femtosecond pulses from a Ti:sapphire laser through a hollowcore photonic crystal fiber," Opt. Lett., vol. 29, no. 11, pp. 1285-1287, Jun. 2004.

[8] J. D. Shephard, P. J. Roberts, J. D. C. Jones, J. C. Knight, and D. P. Hand, "Measuring beam quality of hollow core photonic crystal fibers," J. Lightw. Technol., vol. 24, no. 10, pp. 3761-3769, Oct. 2006.

[9] F. Couny, F. Benabid, and P. S. Light, "Large-pitch kagome-structured hollow-core photonic crystal fiber," Opt. Lett., vol. 31, no. 24, pp. 35743576, Nov. 2006.

[10] S. O. Konorov, C. J. Addison, H. G. Schulze, R. F. B. Turner, and M. W. Blades, "Hollow-core photonic crystal fiber-optic probes for Raman spectroscopy," Opt. Lett., vol. 31, no. 12, pp. 1911-1913, Jun. 2006.

[11] P. S. Light, F. Couny, and F. Benabid, "Low optical insertion-loss and vacuum-pressure all-fiber acetylene cell based on hollow-core photonic crystal fiber," Opt. Lett., vol. 31, no. 17, pp. 2538-2540, Sep. 2006.

[12] T. Murao, K. Saitoh, and M. Koshiba, "Design of air-guiding modified honeycomb photonic band-gap fibers for effectively singlemode operation," Opt. Express, vol. 14, no. 6, pp. 2404-2412, Mar. 2006.

[13] T. Murao, K. Saitoh, and M. Koshiba, "Realization of single-moded broadband air-guiding photonic bandgap fibers," IEEE Photon. Technol. Lett., vol. 18, no. 15, pp. 1666-1688, Aug. 1, 2006.

[14] P. Roberts, F. Couny, H. Sabert, B. Mangan, D. Williams, L. Farr, M. Mason, A. Tomlinson, T. Birks, J. Knight, and P. S. J. Russell, "Ultimate low loss of hollow-core photonic crystal fibres," Opt. Express, vol. 13, no. 1, pp. 236-244, Jan. 2005.

[15] T. Hasegawa, T. Saitoh, D. Nishioka, E. Sasaoka, and T. Hosoya, "Bendinsensitive single-mode holey fiber with SMF compatibility for optical wiring applications," presented at the Eur. Conf. Optical Commun., Rimini, Italy, 2003, Paper We2.7.3.

[16] J. M. Fini, "Design of solid and microstructure fibers for suppression of higher order modes," Opt. Express, vol. 13, no. 9, pp. 3477-3490, May 2005

[17] L. Lavoute, P. Roy, A. Desfarges-Berthelemot, V. Kermène, and S. Février, "Design of microstructured single-mode fiber combining large mode area and high rare Earth ion concentration," Opt. Express, vol. 14, no. 7, pp. 2994-2999, Apr. 2006.

[18] K. Saitoh, N. J. Florous, T. Murao, and M. Koshiba, "Design of photonic band gap fibers with suppressed higher-order modes: Towards the development of effectively single mode large hollow-core fiber platforms," Opt. Express, vol. 14, no. 16, pp. 7342-7352, Aug. 2006.

[19] C. M. Smith, N. Venkataraman, M. T. Gallagher, D. Müller, J. A. West, N. F. Borrelli, D. C. Allen, and K. W. Koch, "Low-loss hollow-core silica/air photonic band-gap fibre," Nature, vol. 424, no. 6949, pp. 657659, Aug. 2003.

[20] R. Amezcua-Correa, N. G. Broderick, M. N. Petrovich, F. Poletti, and D. J. Richardson, "Optimizing the usable bandwidth and loss through core design in realistic hollow-core photonic bandgap fibers," Opt. Express, vol. 14, no. 17, pp. 7974-7985, Aug. 2006.

[21] K. Saitoh and M. Koshiba, "Full-vectorial imaginary-distance beam propagation method based on finite element scheme: Application to photonic crystal fibers," IEEE J. Quantum Electron., vol. 38, no. 7, pp. 927-933, Jul. 2002.

[22] K. Saitoh and M. Koshiba, "Leakage loss and group velocity dispersion in air-core photonic bandgap fibers," Opt. Express, vol. 11, no. 23, pp. 31003109, Nov. 2003.

[23] N. A. Mortensen and M. D. Nielsen, "Modeling of realistic cladding structures for air-core photonic bandgap fibers," Opt. Lett., vol. 29, no. 4, pp. 349-351, Feb. 2004.

[24] K. Saitoh, N. Mortensen, and M. Koshiba, "Air-core photonic bandgap fibers: The impact of surface modes," Opt. Express, vol. 12, no. 3, pp. 394-400, Feb. 2004.

[25] J. West, C. Smith, N. Borrelli, D. Allan, and K. Koch, "Surface modes in air-core photonic band-gap fibers," Opt. Express, vol. 12, no. 8, pp. 14851496, Apr. 2004.

[26] M. A. Duguay, Y. Kokubun, T. L. Koch, and L. Pfeiffer, "Antiresonant reflecting optical waveguides in $\mathrm{SiO}_{2}$-Si multilayer structures," Appl. Phys. Lett., vol. 49, no. 1, pp. 13-15, Jul. 1986.

[27] N. M. Litchinitser, S. C. Dunn, B. Usner, B. J. Eggleton, T. P. White, R. C. McPhedran, and C. M. de Sterke, "Resonances in microstructured optical waveguides," Opt. Express, vol. 11, no. 10, pp. 1243-1251, May 2003

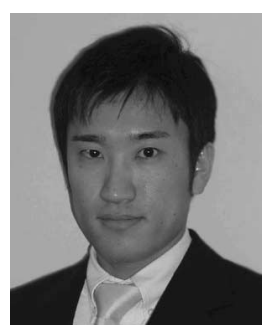

Kunimasa Saitoh (S'00-M'01) was born in Hokkaido, Japan, on July 30, 1973. He received the B.S., M.S., and Ph.D. degrees in electronic engineering from Hokkaido University, Sapporo, Japan, in 1997, 1999, and 2001, respectively.

From 1999 to 2001, he was a Research Fellow of the Japan Society for the Promotion of Science. From 2001 to 2005, he was a Research Associate with Graduate School of Engineering, Hokkaido University. In 2005, he became an Associate Professor with the Graduate School of Information Science and Technology, Hokkaido University. He is with the Division of Media and Network Technologies, Hokkaido University. He has been engaged in research on wave electronics, including lightwaves, surface acoustic waves, and computer-aided design and modeling of guided-wave devices using the finiteelement method, beam propagation method, etc.

Dr. Saitoh is a member of the Institute of Electronics, Information and Communication Engineers (IEICE) and the Optical Society of America. In 1999 and 2002, he was awarded the Excellent Paper Award and the Young Scientist Award, respectively, from the IEICE.

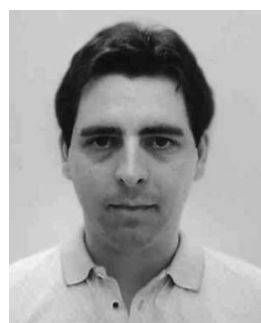

Nikolaos John Florous (M'02) was born in Alonnissos Magnissias, North Sporades, Greece. He received the B.Sc.E.E. degree from the Technical University of Budapest, Budapest, Hungary, in 1998, the M.Sc.E.E degree from the National Technical University of Athens, Athens, Greece, in 2000, and the $\mathrm{Ph} . \mathrm{D}$. degree in electronics and information engineering from Hokkaido University, Sapporo, Japan, in 2006 under the Monbukagakusho Graduate Scholarship nominated from the Government of Japan.

He was with Netcom SA, Greece, from 2001 to 2003 as RF and Microwave Researcher Engineer. He was awarded with the Postdoctoral Fellowship from the Japan Society for the Promotion of Science in April 2006. He is currently appointed with the Division of Media and Network Technologies, Hokkaido University under the supervision of Prof. M. Koshiba. His current research interests include analytical and numerical techniques for electromagnetic wave propagation and scattering in periodic, random, and complex media, modeling of photonic crystal fibers and photonic crystal integrated circuits, optical modeling of artificial metallodielectric nanocomposites (metamaterials), computational electrodynamics, and applied mathematics.

Dr. Florous is a member of the Institute of Electronics, Information, and Communication Engineers of Japan, the Optical Society of America, the American Physical Society, the European Optical Society, the Institution of Electrical Engineers, and the Chinese Institute of Electronics.

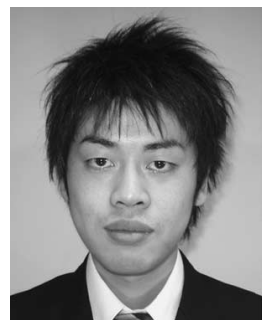

Tadashi Murao was born in Hokkaido, Japan, on December 16, 1982. He received the B.S. degree in media and network technologies from Hokkaido University, Sapporo, Japan, in 2006, where he is currently working toward the M.Sc.Eng. degree.

$\mathrm{He}$ has been engaged in the research on wave electronics, including lightwaves, particularly computeraided design and modeling of photonic band-gap devices using the finite-element method and beam propagation method. He is currently with the Division of Media and Network Technologies, Hokkaido University.

Mr. Murao is a student member of the Institute of Electronics, Information, and Communication Engineers of Japan. 


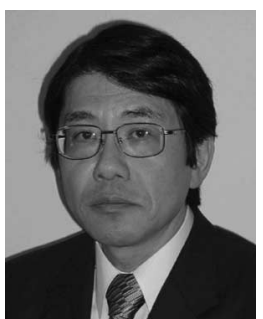

Masanori Koshiba (M'76-SM'87-F'03) was born in Sapporo, Japan, on November 23, 1948. He received the B.S., M.S., and Ph.D. degrees in electronic engineering from Hokkaido University, Sapporo, Japan, in 1971, 1973, and 1976, respectively.

In 1976, he was with the Department of Electronic Engineering, Kitami Institute of Technology, Kitami, Japan. From 1979 to 1987, he was an Associate Professor of electronic engineering with Hokkaido University, where he became Professor in 1987. He is currently with the Division of Media and Network Technologies, Hokkaido University. He has been engaged in research on wave electronics, including microwaves, millimeter-waves, lightwaves, surface acoustic waves, magnetostatic waves, and electron waves, and computeraided design and modeling of guided-wave devices using the finite-element method, boundary element method, beam propagation method, etc. He is an author or coauthor of more than 320 research papers in English and of more than 130 research papers in Japanese both in refereed journals. He is an author of books Optical Waveguide Analysis (McGraw-Hill, 1992) and Optical Waveguide Theory by the Finite Element Method (KTK Scientific/Dordrecht, Kluwer Academic, 1992). He is a coauthor of the books Analysis Methods for Electromagnetic Wave Problems (Artech House, 1990), Analysis Methods for Electromagnetic Wave Problems, Vol. Two (Artech House, 1996), Ultrafast and Ultra-parallel Optoelectronics (Wiley, 1995), and Finite Element Software for Microwave Engineering (Wiley, 1996).

Prof. Koshiba is a Fellow of the Institute of Electronics, Information, and Communication Engineers (IEICE), and is a member of the Institute of Electrical Engineers of Japan, the Institute of Image Information and Television Engineers of Japan, and the Japan Society for Simulation Technology. In 1987, 1997, and 1999, he was awarded the Excellent Paper Awards from the IEICE, in 1998, the Electronics Award from the IEICE-Electronics Society, and, in 2004, the Achievement Award from the IEICE. From 1999 to 2000, he served as President of the IEICE-Electronics Society, and in 2002, he served as Chair of the IEEE Lasers and Electro-Optics Society Japan Chapter. Since 2003, he has been serving on the Board of Directors of the IEICE. 